\title{
Optimization of the Vibro-Impact Capsule System
}

\author{
Yang Liu1,* - Sheikh Islam ${ }^{1}$ - Ekaterina Pavlovskaia ${ }^{2}$ - Marian Wiercigroch ${ }^{2}$ \\ 1 Robert Gordon University, School of Engineering, UK \\ 2 University of Aberdeen, Centre for Applied Dynamics Research, UK
}

\begin{abstract}
Optimization of the vibro-impact capsule system for the best progression is considered in this paper focusing on the choice of the excitation parameters and the shape of the capsule. Firstly, the fastest and the most efficient progressions are obtained through experimental investigations on a novel test bed. Control parameters, the amplitude and the frequency of harmonic excitation, and one of the system parameter, namely the stiffness ratio, are optimized. The experimental results confirm that the control parameters for the fastest progression are not the same as those for the most efficient progression from the energy consumption point of view. Therefore, the capsule system can be controlled either in a speedy mode or in an energy-saving mode depending on the operational requirements. In the second part of the paper, optimization of the capsule shape is studied using computational fluid dynamics (CFD) simulations. Here the aim of achieving the best progression is addressed through minimizing the drag and the lift forces acting on a stationary capsule positioned in the pipe within a fluid flow. The CFD results indicate that both drag and lift forces are dependent on capsule and arc lengths, and finally, an optimized shape of the capsule is obtained.
\end{abstract}

Keywords: capsule system, vibro-impact, experiment, optimization, CFD simulation

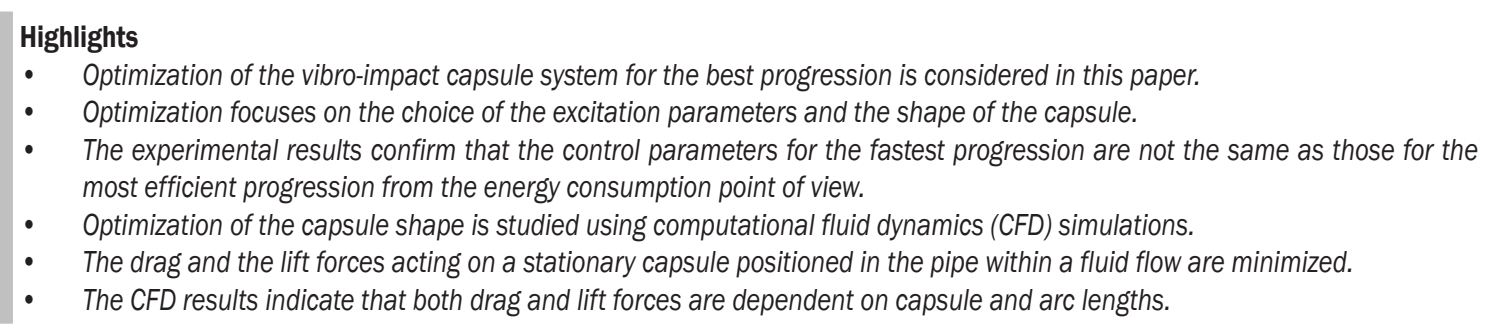

\section{INTRODUCTION}

The inspection and maintenance of pipelines which are usually called "pigging" pose an ongoing challenge that many industries face, including the oil and gas industry [1] and [2]. The use of in-line intelligent pipeline inspection mechanism (known as "pig") is a common method of inspection to determine the condition of oil pipelines [3]. However, developing such a self-propelled mechanism with the accessibility of pipelines in different diameter sizes [4] and [5] is not an easy task, particularly for the design of its driving mechanisms (e.g. legs). Furthermore, a large proportion of pipelines are installed in remote and hostile environments which make traditional inspection mechanisms inaccessible. The vibroimpact capsule system driven by autogenous internal excitation is a promising solution in which there is a growing interest in recent years, e.g. [6] to [9]. Such capsule mechanisms have been demonstrated as an effective way to move in complicated environments, but much less attention has been paid to the optimization of capsule systems. Hence, this paper aims to address this issue focusing on two aspects, i.e. optimization of the capsule dynamics through the appropriate choice of the excitation parameters, and minimizing the resistant forces acting on the capsule in fluid flow via the determination of capsule's optimum geometric parameters.

Optimizations for capsule systems in terms of the average speed of capsule progression have been considered by several researchers. For example, Chernous'ko [10] studied the optimum rectilinear motion of a two-mass system to obtain its maximum mean velocity. Li et al. [11] proposed a minimum energy solution for an internal mass-driven capsule system. In [12], the dynamics of a mobile capsule system containing a movable internal mass was optimized with respect to its maximal average steadystate velocity. However, there is less published work which studied optimizations for such capsule systems experimentally. In [9], using a mathematical model of the capsule system, Liu et al. found that the parameters of the vibro-impact capsule system for the best progression and for the minimum energy consumption were different. To consider the capsule motion in different environmental conditions, vibroimpact responses of the capsule system with various friction models were investigated in [13]. In [14], a position feedback control method suitable for 
dealing with chaos control and coexisting attractors was studied for enhancing the desirable forward and backward capsule motion. Recently, the dynamical response of the capsule system was studied by means of path-following techniques in [15] focusing on two practical problems which were maximizing the rate of progression and directional control of the system by following a typical period-1 trajectory. In this paper, the experimental verification of the theoretical finding from [9] will be presented, where the recently designed test bed proposed in [16] is used for this experimental study. In [16], the comparisons between the experiments and numerical simulations were presented showing a good agreement, and the conducted bifurcation analysis indicated that the behaviour of the system was mainly periodic and that a fine tuning of the control parameters can significantly improve the performance of the system. Differently, optimal control parameters with respect to capsule average speed and power efficiency will be identified experimentally in this paper through varying the frequency and amplitude of external excitation and stiffness ratio.

As the capsule geometry plays a significant role in the dynamic behaviour of the system in the fluid, computational fluid dynamics (CFD) has been adopted by many researchers to investigate capsule movement in pipeline. In [17], Feng et al. studied two-dimensional simulations of the motion of elliptic capsules carried by a Poiseuille flow in a channel. Khalil et al. [18] studied the pressure distribution around a capsule by using a three-dimensional steadystate turbulent flow CFD model. Borregales et al. [19] investigated the pigging process inside pipeline through two-dimensional transient CFD simulations, and found that sliding at constant velocity allows pig to move easily inside a straight pipe. Li et al. performed unsteady turbulent flow CFD simulations of a single coal log by using a dynamic mesh method in [20]. In this paper, CFD modelling will be carried out for optimizing capsule shape (e.g. capsule and arc lengths) in order to obtain the minimum drag and lift forces in a dynamic flow condition. CFD simulations were carried out considering that the capsule moves in the same direction as the fluids flow. So the findings in this paper could be used for prototype design and fabrication.

The rest of the paper is organized as follows. In Section 1, the experimental apparatus and the mathematical model of the vibro-impact capsule system are briefly introduced, and then experimental optimization of the capsule dynamics is presented. In Section 2, the CFD analysis is described and the results are discussed. Finally, some conclusions are drawn in Section 3.

\section{DYNAMICS AND ENERGY OPTIMIZATIONS}

\subsection{Experimental Apparatus}

To verify the theoretical finding obtained in [9], the test bed [16] shown in Fig. 1a is used in this study. The experimental rig consists of a linear DC servomotor mounted on a base frame connected with a standing frame which holds a support spring with an adjustable stiffness $k_{2}$. The motor has a movable rod with the mass $m_{1}$ harmonically excited with a desired frequency $\omega$ and amplitude $p_{d}$ through the electro-magnetic fields generated by the coils within the motor. Although a nonlinear resistance force keeps the rod in place when the motor is switched on, we assume that this force could be linearized around the working point and characterised by constant coefficients $k_{1}$ and $c$ multiplied by the displacement and velocity, respectively. A gap $\delta$ exists between the rod and the support spring, and the rod contacts with the support spring when their relative displacement is
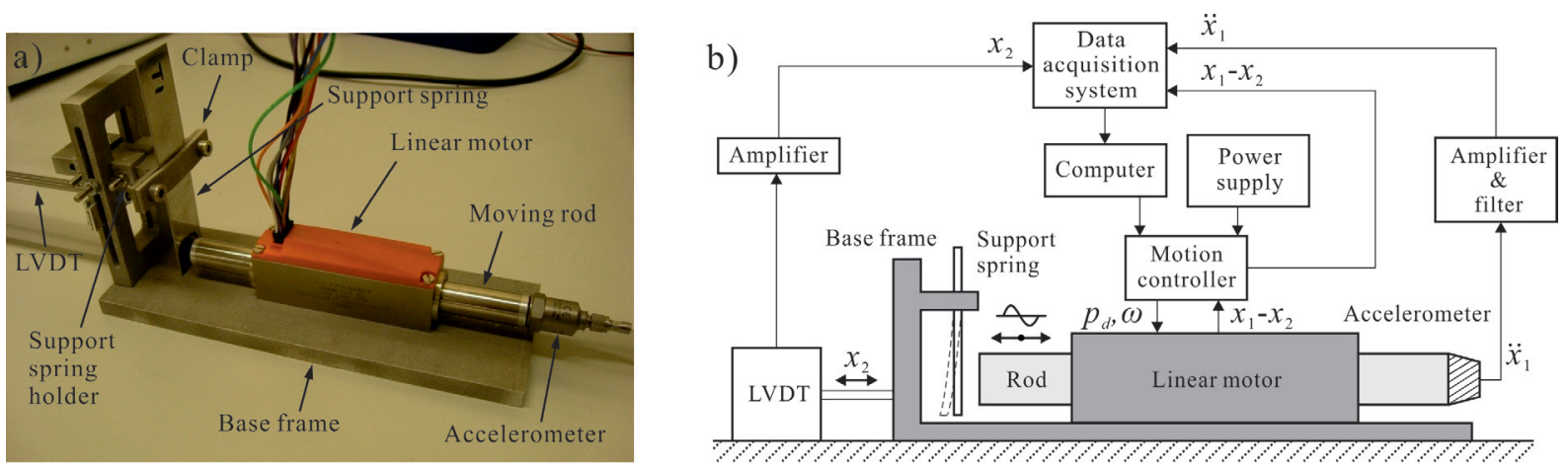

Fig. 1. a) Photograph of the test bed; and b) schematic of the experimental setup [16] 
larger or equals to the gap. A detailed description of its physical parameter identification can be found in [16].

A schematic of the experimental setup is shown in Fig. 1b, where the absolute displacement of the rod is $x_{1}$, and the absolute displacement of the base frame is $x_{2}$ which is measured by a linear variable differential transformer (LVDT) displacement transducer. The relative displacement of the rod and the base frame $x_{1}-x_{2}$ is measured via three hall sensors within the motor. The acceleration of the rod is obtained using an accelerometer mounted directly on the rod.

\subsection{Non-Dimensional Equations of Motion}

Similar to the system described in [9], [13] and [14], in this study the vibro-impact capsule is modelled as a two degrees-of-freedom dynamical system depicted in Fig. 2, where a movable internal mass $m_{1}$ is driven by a harmonic force with amplitude $p_{d}$ and frequency $\omega$ generated by a linear actuator. The actuator contains a movable part connected to the internal mass and a fixed part mounted on the rigid capsule $m_{2}$. We simplify the model of the actuator here and represent the interaction between the internal mass and the capsule by using a linear spring with stiffness $k_{1}$ and a viscous damper with damping coefficient $c . x_{1}$ and $x_{2}$ represent the absolute displacements of the internal mass and the capsule, respectively. The internal mass contacts a weightless plate connected to the capsule by a secondary linear spring with stiffness $k_{2}$ when the relative displacement $x_{1}-x_{2}$ is larger or equals to the gap $\delta$. When the force acting on the capsule exceeds the threshold of the dry friction force $f_{s}$ between the capsule and the supporting environmental surface, bidirectional motion of the capsule will occur, and the dynamic friction force $f_{d}$ will be applied to the capsule. As the considered system operates in bidirectional stick-slip phases, a detailed consideration of these phases can be found in [9].

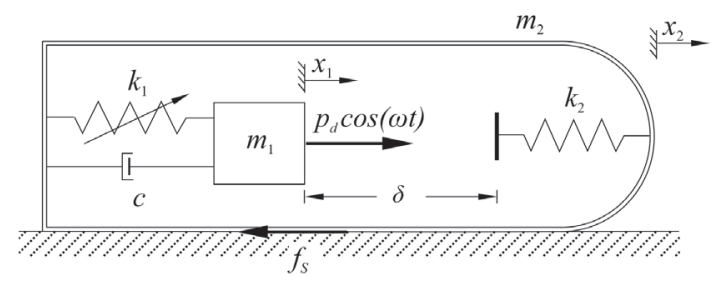

Fig. 2. Physical model of the vibro-impact capsule system

In order to re-scale the capsule dynamics and extend the experimental findings to a general vibroimpact driven system, we introduce the following non-dimensional variables:

$$
\begin{gathered}
\tau=\omega_{0} t, \quad \bar{x}_{i}=\frac{k_{1}}{f_{s}} x_{i}, \quad \bar{y}_{i}=\frac{\mathrm{d} \bar{x}_{i}}{\mathrm{~d} \tau}=\frac{k_{1}}{\omega_{0} f_{s}} \dot{x}_{i}, \\
\dot{\bar{y}}_{i}=\frac{\mathrm{d} \bar{y}_{i}}{\mathrm{~d} \tau}=\frac{k_{1}}{\omega_{0}^{2} f_{s}} \ddot{x}_{i}, \quad \bar{f}_{d}=\frac{f_{d}}{f_{s}},
\end{gathered}
$$

and parameters:

$$
\begin{gathered}
\omega_{0}=\sqrt{\frac{k_{1}}{m_{1}}}, \quad \bar{\omega}=\frac{\omega}{\omega_{0}}, \quad \alpha=\frac{p_{d}}{f_{s}}, \quad \zeta=\frac{c}{2 m_{1} \omega_{0}}, \\
\bar{\delta}=\frac{k_{1}}{f_{s}} \delta, \quad \beta=\frac{k_{2}}{k_{1}}, \quad \gamma=\frac{m_{2}}{m_{1}},
\end{gathered}
$$

where $i=1,2$. Then the equations of motion can be written in a dimensionless form as (see [9] for the detailed derivations):

$$
\begin{aligned}
\dot{\bar{x}}_{1}= & \bar{y}_{1}, \\
\dot{\bar{y}}_{1}= & \alpha \cos (\bar{\omega} \tau)+\left(\bar{x}_{2}-\bar{x}_{1}\right)+2 \zeta\left(\bar{y}_{2}-\bar{y}_{1}\right) \\
& -h_{1} \beta\left(\bar{x}_{1}-\bar{x}_{2}-\bar{\delta}\right), \\
\dot{\bar{x}}_{2}= & \bar{y}_{2}\left[h_{2}\left(1-h_{1}\right)+h_{3} h_{1}\right], \\
\dot{\bar{y}}_{2}= & {\left[h_{2}\left(1-h_{1}\right)+h_{3} h_{1}\right]\left[-\bar{f}_{d}-\left(\bar{x}_{2}-\bar{x}_{1}\right)\right.} \\
& \left.-2 \zeta\left(\bar{y}_{2}-\bar{y}_{1}\right)+h_{1} \beta\left(\bar{x}_{1}-\bar{x}_{2}-\bar{\delta}\right)\right] / \gamma,
\end{aligned}
$$

and the auxiliary Heaviside functions $h_{i}(i=1, \ldots, 3)$ are given by

$$
\begin{aligned}
h_{1} & =h\left(\bar{x}_{1}-\bar{x}_{2}-\bar{\delta}\right), \\
h_{2} & =h\left(\left|\left(\bar{x}_{2}-\bar{x}_{1}\right)+2 \zeta\left(\bar{y}_{2}-\bar{y}_{1}\right)\right|-1\right), \\
h_{3} & =h\left(\left|\left(\bar{x}_{2}-\bar{x}_{1}\right)+2 \zeta\left(\bar{y}_{2}-\bar{y}_{1}\right)\right|\right. \\
& \left.-\beta\left(\bar{x}_{1}-\bar{x}_{2}-\bar{\delta}\right)-1\right) .
\end{aligned}
$$

Based on the analysis conducted in [13], where four various friction models were used to describe the motion of the capsule in different environments, the friction of the experimental test bed in [16] was found to be well approximated by the Coulomb Stribeck model given by:

$$
\bar{f}_{d}=\left(1+e^{-\frac{\left|\dot{\bar{x}}_{2}\right|}{v_{s}}}\right) \operatorname{sign}\left(\dot{\bar{x}}_{2}\right),
$$

where $v_{s}$ is the non-dimensional Stribeck velocity.

\subsection{Experimental Optimization}

In order to optimise the performance of the system, we introduce the average progression of the capsule system over one period of the external excitation $T_{a}=2 \pi / \bar{\omega}$, 


$$
P_{\text {avg }}=\frac{1}{T_{a}}\left[\bar{x}_{2}\left(T_{a}\right)-\bar{x}_{2}(0)\right],
$$

and the power efficiency which is the ratio of the capsule progression to the work done by the external excitation over the period $T_{a}$,

$$
E_{\text {avg }}=\frac{\bar{x}_{2}\left(T_{a}\right)-\bar{x}_{2}(0)}{\int_{0}^{T_{a}} \alpha \cos (\bar{\omega} \tau) \bar{y}_{1}(\tau) \mathrm{d} \tau} .
$$

After non-dimensionalizing the experimental results, both performance indices, $P_{\text {avg }}$ and $E_{\text {avg }}$ were calculated in order to determine the optimum control parameters.

Optimizations of the frequency of excitation $\bar{\omega}$ for $\beta=8.92$ and $\beta=13.35$ are presented in Fig. 3 and 4, respectively. The optimal frequency of the fastest forward progression for $\beta=8.92$ is $\bar{\omega}=0.29$, and the optimal frequency for $\beta=13.35$ is $\bar{\omega}=0.3$. It is worth noting that the optimal progression for $\beta=8.92$ is chaotic including both forward and backward motions in every period of excitation, while only periodic forward motion exists for the optimal progression for $\beta=13.35$ as shown in the blow-up window of Fig. 4.

Optimization of the amplitude of excitation $p_{d}$ for $\beta=13.35$ is $\bar{\omega}=0.61$, and $\bar{\delta}=0.34$ is presented in Fig. 5 where both forward and backward progressions were recorded. It can be seen from the figure that the best forward progression is achieved at $\alpha=0.27$ and the best backward progression is obtained at $\alpha=0.41$. This experimental result reveals that the largest amplitude of excitation will not generate the fastest

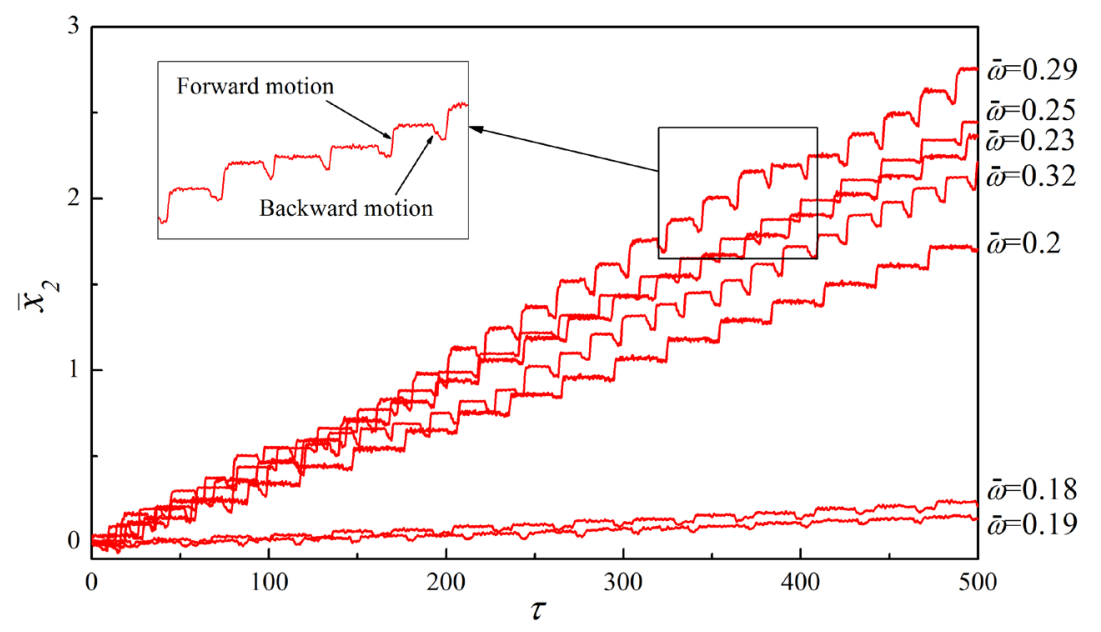

Fig. 3. Time histories of the capsule displacement, $\bar{x}_{2}$ obtained experimentally by varying the frequency of excitation $\bar{\omega}$ at $\gamma=4.82, \zeta=0.16, \beta=8.92, \alpha=0.97$, and $\bar{\delta}=0.52$

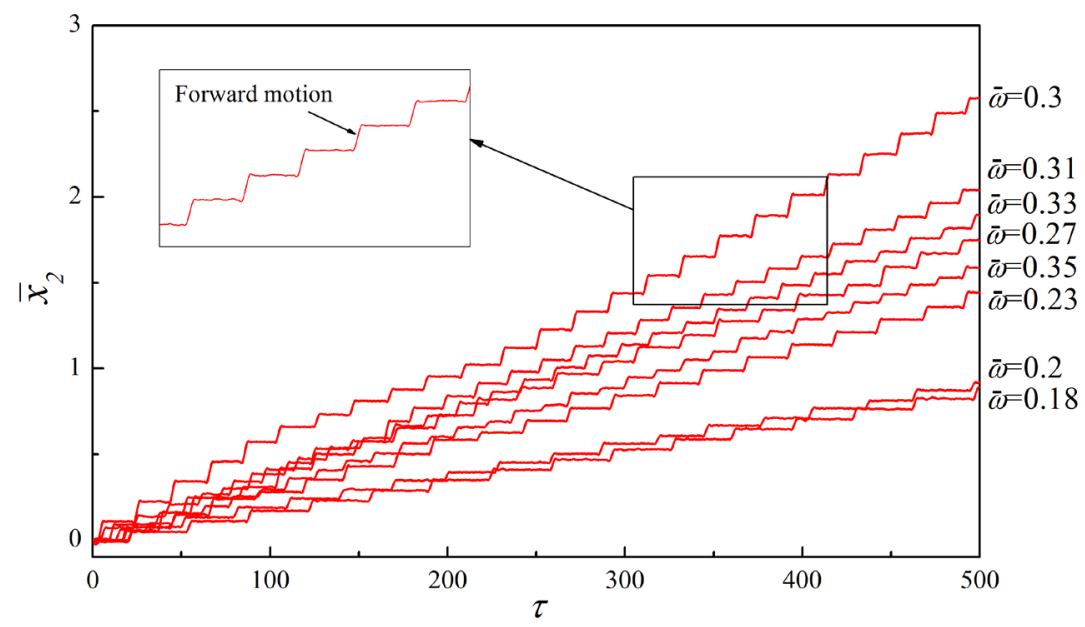

Fig. 4. Time histories of the capsule displacement, $\bar{x}_{2}$ obtained experimentally by varying the frequency of excitation $\bar{\omega}$ at $\gamma=4.82, \zeta=0.16, \beta=13.35, \alpha=0.97$, and $\bar{\delta}=0.52$ 


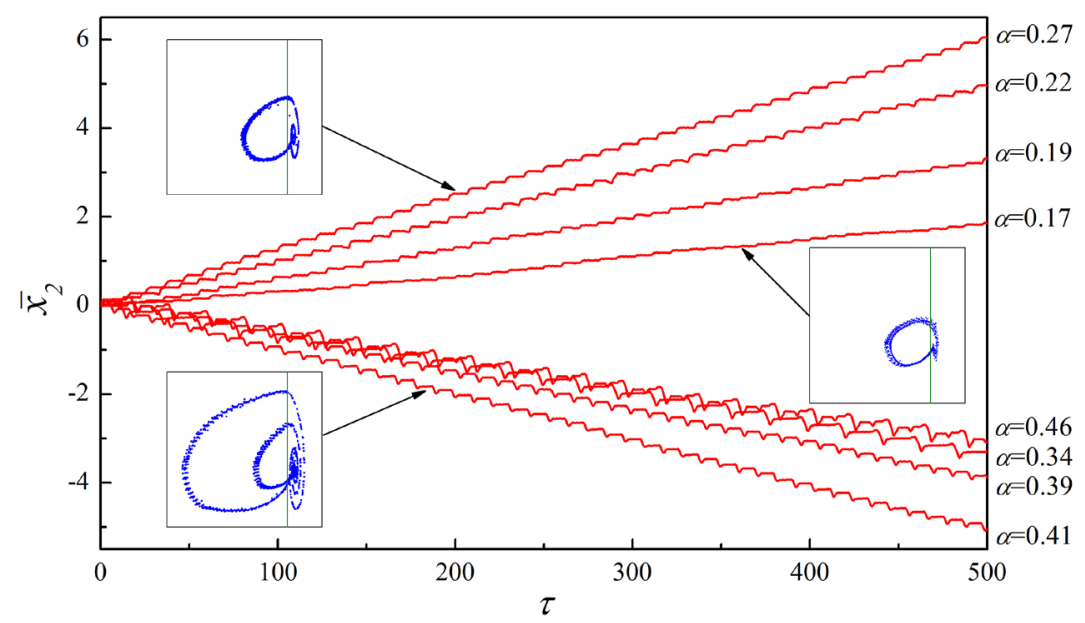

Fig. 5. Time histories of the capsule displacement, $\bar{x}_{2}$ obtained experimentally by varying the amplitude of excitation $\alpha$ at $\gamma=4.82, \zeta=0.16, \beta=13.35, \bar{\omega}=0.61$, and $\bar{\delta}=0.34$; phase trajectories are presented in blow-up windows
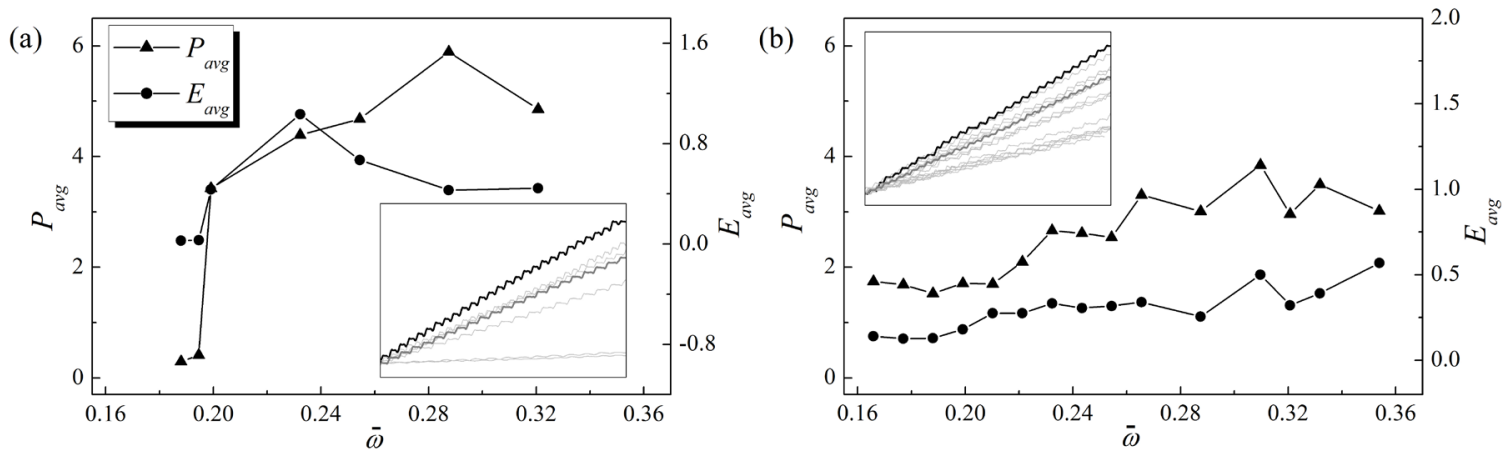

Fig. 6. Average progress, $P_{\text {avg }}$ and energy efficiency, $E_{\text {avg }}$ obtained experimentally by varying the frequency of excitation, $\bar{\omega}$ at a) $\beta=8.92$, and b) $\beta=13.35, \gamma=4.82, \alpha=0.97$, and $\bar{\delta}=0.514$

capsule progression, thereby a proper selection of optimal parameters should be considered for different configurations of the system.

Fig. 6 presents the performance indices $P_{a v g}$ and $E_{\text {avg }}$ recalculated for the experimental results shown in Figs. 3 and 4. As can be seen from Fig. 6a, the fastest progression is $\bar{\omega}=0.29$ and the most efficient progression is $\bar{\omega}=0.23$ for $\beta=8.92$. As the stiffness ratio $\beta$ increases to 13.35 , the fastest and the most efficient progressions are recorded at $\bar{\omega}=0.31$ and $\bar{\omega}=0.35$, respectively. All the capsule displacements in time histories are displayed in the embedded windows where the fastest progression is indicated by black line, the most efficient progression is shown by grey line and the rest of displacements are denoted by light grey lines. It can be seen that more energy is spent per period during the fastest motion due to spell of backward motion. This causes the fastest progression shown by black line less efficient comparing with the one indicated by grey line.
Fig. 7 shows a series of experiments by varying the amplitude of excitation. In order to compare the capsule speed in one direction, we chose a set of control parameters which has forward progression only. It can be seen from the figure that both the fastest and the most efficient progressions were achieved at $\alpha=0.29$, so only one capsule progression marked by black line is presented in the embedded window.

The experimental results as a function of stiffness ratio are shown in Fig. 8. As $k_{1}$ was kept as a constant throughout the experiments, the variation of the stiffness ratio were implemented by changing the length of the support spring, i.e. the stiffness of the support spring $k_{2}$. As can be observed from the figure, the fastest and the most efficient progressions were achieved at $\beta=16.1$ and $\beta=8.92$, respectively. It is also noted that when the stiffness ratio is small, both performance indices are much better than the ones for large stiffness ratio. 


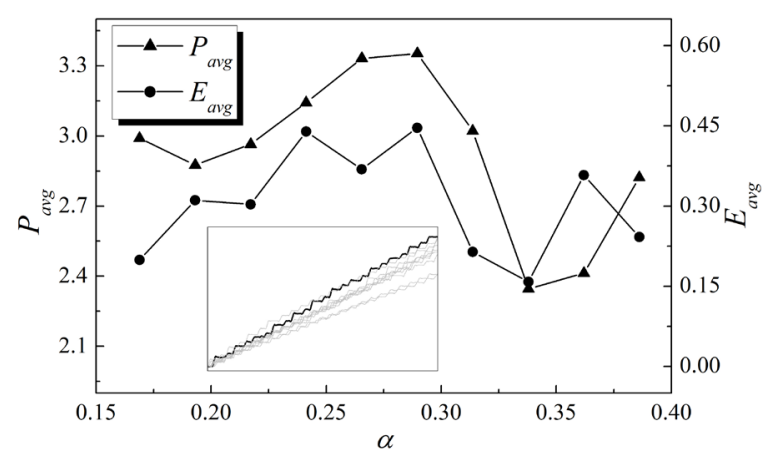

Fig. 7. Average progress, $P_{\text {avg }}$ and energy efficiency, $E_{\text {avg }}$ obtained experimentally by varying the amplitude of excitation, $\alpha$ at $\beta=13.35, y=4.82, \bar{\omega}=0.277$, and $\bar{\delta}=0.514$

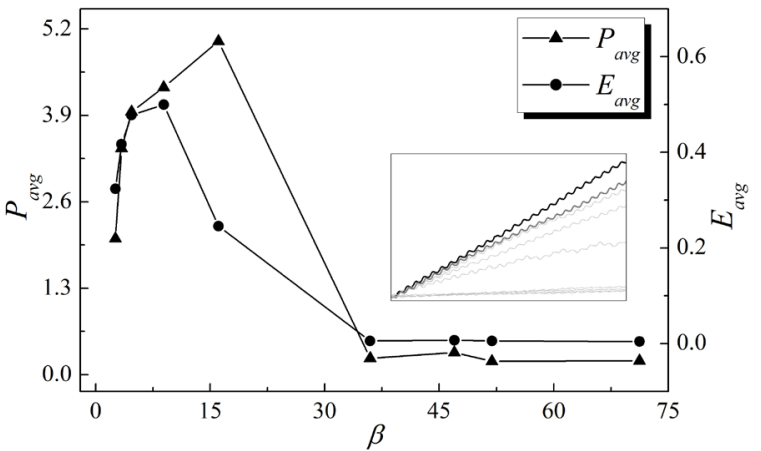

Fig. 8. Average progress, $P_{\text {avg }}$ and energy efficiency, $E_{\text {avg }}$ obtained experimentally by varying the stiffness of the support spring, $\beta$ at $y=4.82, \alpha=0.97 \bar{\omega}=0.304$, and $\bar{\delta}=0.514$

The results of the presented experimental study allow one to obtain the optimal excitation forcing in order to drive the capsule system either in fastest or the most efficient ways. Although the experiments were conducted in air, our mathematical modelling [13] indicates that similar results could be obtained if the capsule is placed on the heavily lubricated surface. However, if the capsule is fully submerged and moving in the fluid, there will be lift and drag forces creating the resistance to the capsule motion. Therefore, in the next section, we will consider optimization of the stationary capsule shape using CFD approach.

\section{CFD OPTIMIZATION}

\subsection{CFD Model}

A two-dimensional steady-state model of the vibroimpact capsule system inside a pipe was developed in this section in order to investigate the effect of shape of the capsule on the hydrodynamic coefficients. The purpose of the study was to optimize capsule shape for reducing resistance force from the fluid. Here one of the key assumptions was that the test bed used in Section 1 is fully enclosed in an outer capsule. Although in real applications, the capsule will be moving along the pipeline within the fluid flow, in the first approximation, the stationary capsule was considered in this study.

Table 1. Model parameters for $2 D$ simulation

\begin{tabular}{lll}
\hline Model Parameters & Symbol & Value \\
\hline Capsule length & $L_{c}$ & $118 \mathrm{~mm}$ \\
\hline Capsule diameter & $D_{c}$ & $80 \mathrm{~mm}$ \\
\hline Arc length & $R_{R H S}$ & $40 \mathrm{~mm}$ \\
\hline Pipe internal diameter & $D_{p}$ & $140 \mathrm{~mm}$ \\
\hline Pipe length & $L_{p}$ & $2000 \mathrm{~mm}$ \\
\hline Pipe/Capsule clearance & $L_{c l e a r}$ & $1 \mathrm{~mm}$ \\
\hline Inlet velocity & $V_{i}$ & $0.5 \mathrm{~m} / \mathrm{s}$ \\
\hline Outlet pressure & $P_{o}$ & $0 \mathrm{MPa}$ \\
\hline
\end{tabular}

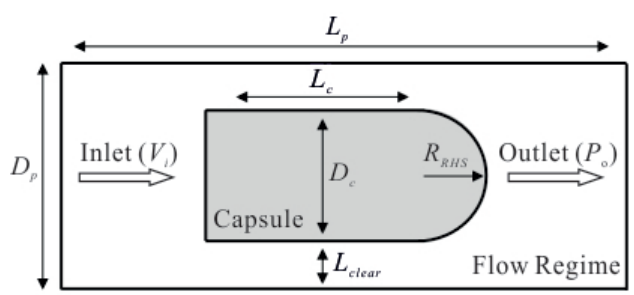

Fig. 9. 2D capsule simulation layout and boundary conditions

Design of Experiment (DOE) technique is implemented to explore the effects of multi variable variation on the reduction of drag forces, and to improve the efficiency of the vibro-impact mechanism. Two-dimensional modelling approach was selected to reduce the complexity of the modelling process and costly simulation time. Fig. 9 shows the two-dimensional numerical model with boundary conditions, and the physical dimensions of the computational domain given in Table 1 are based on the dimension of our original prototype.

The assumptions made in solving the 2D computational model are given as follows.

- The optimization study is performed under steady state condition of the capsule, i.e. the operating conditions and the capsule position inside the pipe do not change with time.

- The capsule is operated under isothermal conditions.

- A pressure outlet condition is assumed and no slip condition is applied to the external walls.

- The flow is turbulent based on the fluid properties and boundary conditions. 
- A small gap is assumed between the capsule bottom and the inner wall of the pipe in order to reduce the high frictional resistance due to contact.

As the flow condition was calculated to be turbulent, the $k-\varepsilon$ turbulent model [21] was adopted for the study. The model includes the turbulent kinetic energy represented by the turbulent viscosity:

$$
\mu_{t}=\rho C_{\mu} k^{2} / \varepsilon
$$

and the turbulent kinetic energy,

$$
\frac{\partial k}{\partial t}+U_{j} \frac{\partial k}{\partial x_{j}}=\tau_{i j} \frac{\partial U_{i}}{\partial x_{j}}-\varepsilon+\frac{\partial}{\partial x_{j}}\left[\left(v+v_{t} / \sigma_{k}\right) \frac{\partial k}{\partial x_{j}}\right],
$$

and the turbulent dissipation expressed as:

$$
\begin{gathered}
\frac{\partial \varepsilon}{\partial t}+U_{j} \frac{\partial \varepsilon}{\partial x_{j}} \\
=C_{\varepsilon 1} \frac{\varepsilon}{k} \tau_{i j} \frac{\partial U_{i}}{\partial x_{j}}-C_{\varepsilon 2} \frac{\varepsilon^{2}}{k}+\frac{\partial}{\partial x_{j}}\left[\left(v+\frac{v_{t}}{\sigma_{k}}\right) \frac{\partial \varepsilon}{\partial x_{j}}\right] .
\end{gathered}
$$

The flow domain was created as a rectangular cross-section of the pipeline with the capsule position at approximately halfway between the inlet and the outlet. The left face and the right face of the pipeline were defined as being a velocity inlet and pressure outlet, respectively. The upper face of pipeline, lower pipeline and the capsule were defined as standard walls.

\subsection{Computational Procedure and Modelling Parameters}

The governing equations were solved using the finite volume method for CFD solver (ANSYS FLUENT) which is based on the SIMPLE (Semi Implicit Method for Pressure Linked Equation) algorithm. The relative error is considered less than $10^{-3}$ for convergence. The multi-factor DOE optimization technique was utilized in simulations in order to establish the most suitable design parameters for the capsule. Two parameters, capsule length $L_{c}$ (between 118 and $150 \mathrm{~mm}$ ) and arc length $R_{R H S}$ (between 40 and $80 \mathrm{~mm}$ ) were considered as the factors for each simulation, and the best design for reduced drag force allowing the capsule to move faster was obtained. These measurement regions were selected based on the design constraints of the vibro-impact mechanism of the capsule. The goal of the optimization is to improve the existing capsule design such that the hydrodynamic coefficients are made as low as possible. Since two design parameters were weighed against each other in each study, three dimensional response output plots of the results were created to show which parameters allow the most optimal operation. The goal-driven optimization has created 50 sample simulations, and 3 potential candidates were chosen by using the objective function. The computational domain has been meshed with 106818 elements including close wall biasing. A grid sensitivity test using over 200000 cells has proven that the grid size is sufficient to provide grid independency. In addition, each simulation took approximately 1800 iterations with an average convergence time of 20 minutes, and velocity contours, streamlines, pressure contours and response surface were achieved through ANSYS Post processing.

\subsection{Results and Discussion}

This section outlines and discusses the results for the DOE study which investigates the effects of the capsule length $L_{c}$ and the arc length $R_{R H S}$ on the drag and the lift coefficients. Fig. 10 presents the response
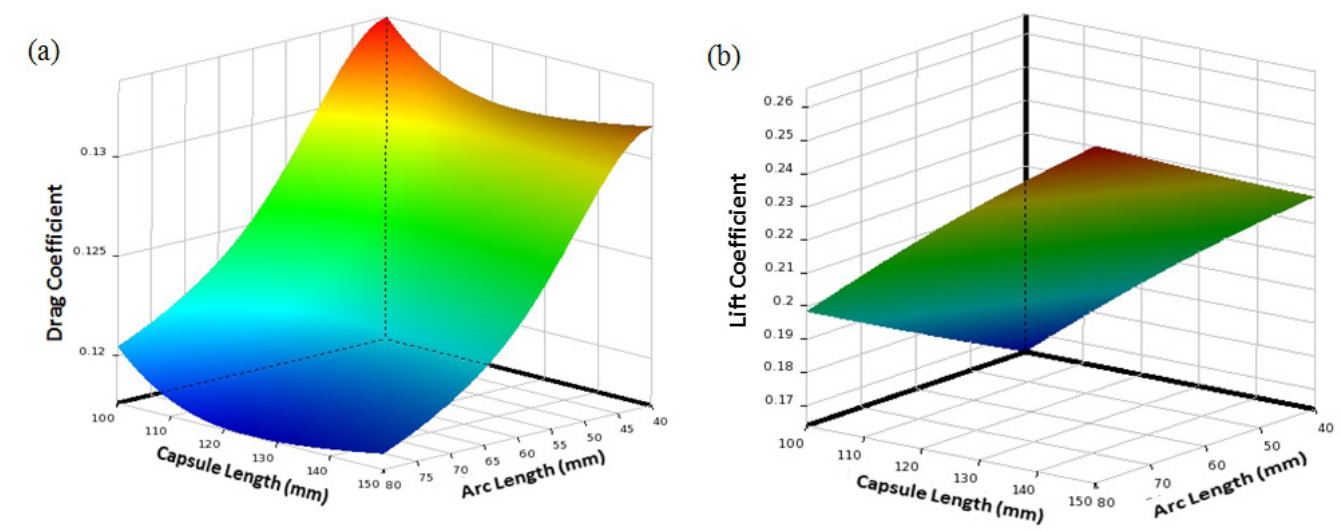

Fig. 10. Response surfaces for a) drag and b) lift coefficients 
(a)

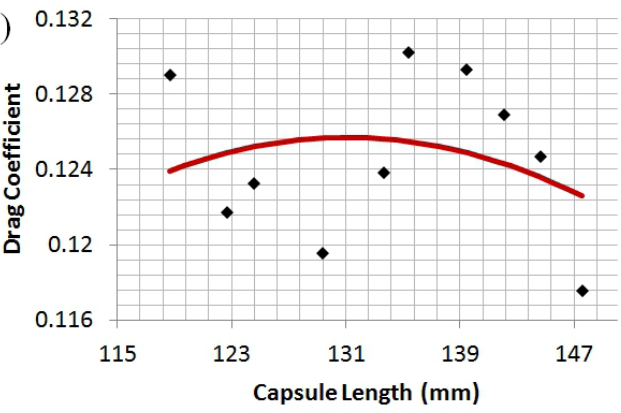

(b)

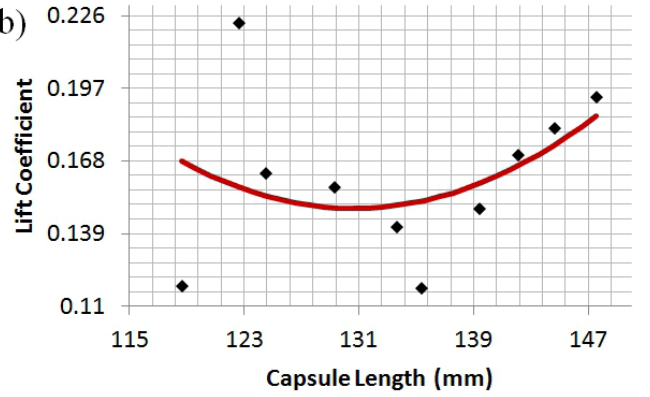

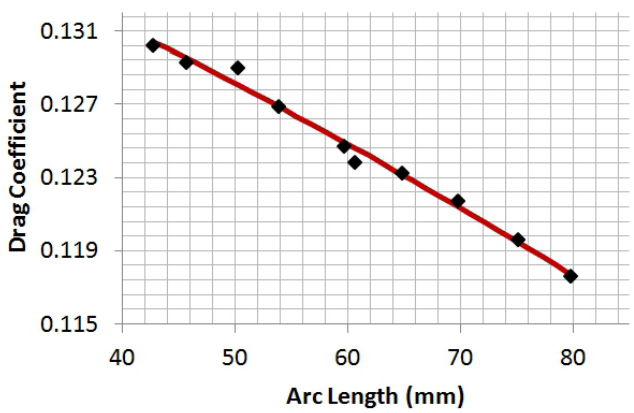

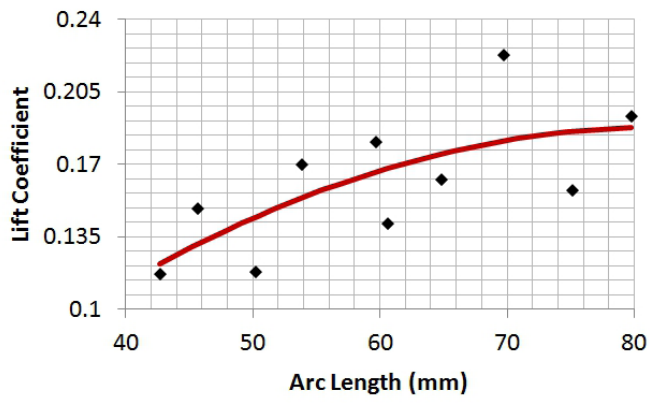

Fig. 11. Effect of capsule and arc lengths on a) drag and b) lift coefficients; coefficients are marked by diamonds and linear fits are shown by lines

surfaces for both coefficients. It can be seen from the figure that the minimum drag coefficient is achieved when both arc and capsule lengths increase. Fig. 11 shows the parametric correlation between the drag and the lift coefficients with the capsule and the arc lengths. The plots were created using the results obtained from 10 design points out of 50 analysed. The results confirm again that both coefficients are dependent on the arc and the capsule lengths.

Fig. 12 presents the pressure contours, the velocity contours, and the streamlines for the original and the optimum capsule designs. Comparing both designs, the downstream nose cone has been enlarged and the capsule length has been increased for the optimum design. Pressure and velocity contours show a slight reduction in passing fluid pressure and velocity at downstream. This small variation reduces the drag coefficient, and hence will improve the motion of the capsule in pipe.

The goal-driven optimization studies identified three best candidates for the minimum drag and lift forces, and the optimized design variables for the best candidate of capsule design are given in Table 2. A graphic comparison between the original and the optimum designs is presented in Fig. 13.
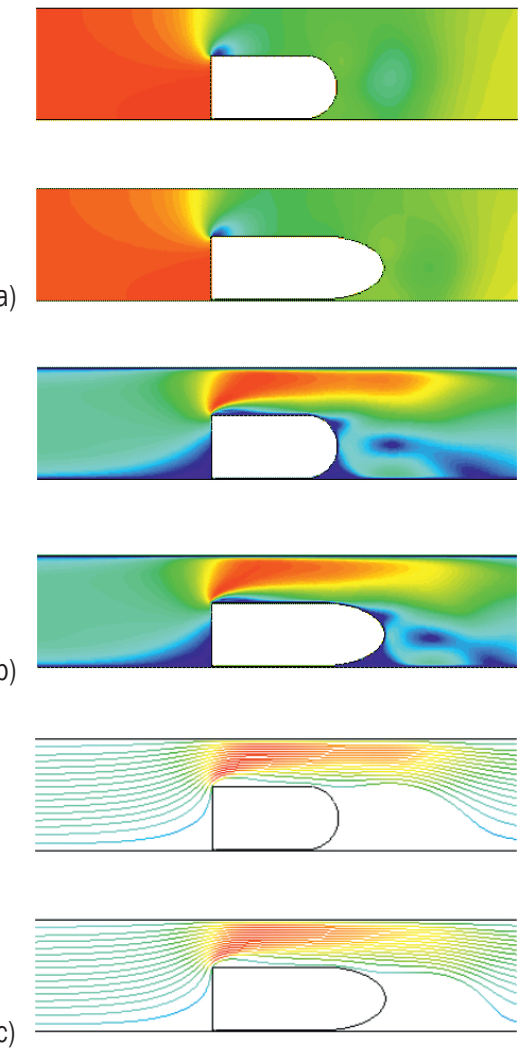

Fig. 12. Plots of a) pressure contours, $b$ ) velocity contours, and c) streamlines for the original (upper) and the optimum (lower) designs 
Table 2. Optimized capsule measurements

\begin{tabular}{lccc}
\hline Model parameter & Symbol & Original value & Optimum value \\
\hline Capsule length & $L_{c}$ & $118 \mathrm{~mm}$ & $138.16 \mathrm{~mm}$ \\
\hline Arc length & $R_{R H S}$ & $40 \mathrm{~mm}$ & $79.15 \mathrm{~mm}$ \\
\hline
\end{tabular}

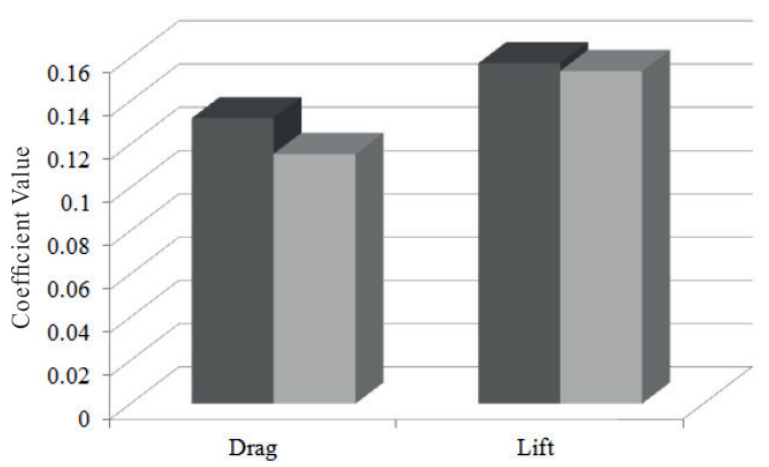

Fig. 13. Comparison of the original (darker) and the optimum (lighter) capsule designs

\section{CONCLUSIONS}

Optimization of the vibro-impact capsule system was studied in this paper experimentally using a novel test bed and numerically through CFD simulations. The paper is focused on addressing two optimization issues, namely the choice of the excitation force parameters for driving the capsule and the choice of the capsule shape to minimize the resistance force. The aim in both cases is to obtain the best progression of the capsule system.

Optimization for the progression of the capsule system was carried out experimentally by varying the frequency and the amplitude of excitation. The experimental results revealed that the best progression of the system for stiffness ratio $\beta=8.92$ was achieved at the excitation frequency $\bar{\omega}=0.29$ when the motion of the capsule was chaotic including both forward and backward motions in every period of excitation, while only periodic forward motion exists for $\beta=13.35$ and the optimal frequency is $\bar{\omega}=0.3$. Our comparisons on the average progression $P_{a v g}$ and the power efficiency $E_{\text {avg }}$ have confirmed that the control parameters for the fastest progression are not the most efficient ones, so the capsule system can be controlled either in a speedy mode or an energy-saving mode depending on the requirement of operation.

The optimum capsule geometric parameters for the best progression were identified through minimizing the drag and the lift forces using CFD simulation by assuming that a stationary "ideal" capsule was positioned in the pipe with fluid flow.
Here we assumed that the experimental test bed was encapsulated and only an outer capsule was considered for CFD simulation. An optimum design could be achieved by minimizing the drag and lift resistance forces acting on the capsule. The CFD results indicate that both drag and lift forces are dependent on the capsule and the arc lengths. Finally, an optimum set of capsule and arc lengths was obtained and the improvement was demonstrated using a histogram representation.

Parametric studies for this optimum design through three-dimensional modelling and time dependent simulations would be the future work which could provide a comprehensive understanding of how the capsule interacts with the fluids and accurately predicts the performance of the capsule in real scenarios. Consideration of the moving capsule in the fluid flow would be the next step in our modelling for the shape optimization. Also, creating a model where the capsule is fully submerged and moving in the fluid with proper lift and drag forces acting on the capsule would be an interesting study which would allow refining the driving force optimization strategy. The uncertainty of the input parameters, environmental friction and fluid velocity will be investigated in the dynamical model and the CFD analysis in the future work, respectively, and the findings will be reported in due course.

\section{ACKNOWLEDGMENTS}

Dr. Yang Liu would like to acknowledge the financial support for the Small Research Grant (31841) by the Carnegie Trust for the Universities of Scotland.

\section{REFERENCES}

[1] Shukla, A., Karki, H. (2016). Application of robotics in onshore oil and gas industry - a review Part I. Robotics and Autonomous Systems, vol. 75, p. 490-507, D0l:10.1016/j. robot.2015.09.012.

[2] Shukla, A., Karki, H. (2016). Application of robotics in onshore oil and gas industry - a review Part II. Robotics and Autonomous Systems, vol. 75, p. 508-524, D0l:10.1016/j. robot.2015.09.013.

[3] Russell, D., Errington, N. (2012). Assessing the effectiveness of pipeline cleaning programs. Pipeline \& Gas Journal, vol. 239, no. 6.

[4] Zhang, Y., Jiang, S., Zhang, X., Ruan, X., Guo, D. (2011). A variable-diameter capsule robot based on multiple wedge effects. IEEE-ASMETransactions of Mechatronics, vol. 16, no. 2, p. 241-254, DOI:10.1109/TMECH.2009.2039942.

[5] Park, J., Hyun, D., Cho, W., Kim, T., Yang, H. (2011). Normalforce control for an in-pipe robot according to the inclination of 
pipelines. IEEE Transactions on Industrial Electronics, vol. 58, no. 12, p. 5304-5310, D0I:10.1109/TIE.2010.2095392.

[6] Nagy, Z., Leine, R.I., Frutiger, D.R., Glocker, C., Nelson, B.J. (2012). Modeling the motion of microrobots on surfaces using nonsmooth multibody dynamics. IEEE Transaction on Robotics, vol. 28, no. 5, p. 1058-1068, Dol:10.1109/ TR0.2012.2199010.

[7] Carta, R., Sfakiotakis, M., Pateromichelakis, N., Thoné, J., Tsakiris, D.P., Puers, R. (2011). A multi-coil inductive powering system for an endoscopic capsule with vibratory actuation. Sensors and Actuators A: Physical, vol. 172, no. 1, p. 253 258, D0l:10.1016/j.sna.2011.03.036.

[8] Sfakiotakis, M., Pateromichelakis, N., Tsakiris, D. (2014). Vibration-induced frictional reduction in miniature intracorporeal robots. IEEE Transactions on Robotics, vol. 30, p. 1210-1221, D0I:10.1109/TR0.2014.2334931.

[9] Liu, Y., Wiercigroch, M., Pavlovskaia, E., Yu, H. (2013). Modelling of a vibro-impact capsule system. International Journal of Mechanical Sciences, vol. 66, p. 2-11, DOI:10.1016/j.ijmecsci.2012.09.012.

[10] Chernous'ko, F. (2002). The optimum rectilinear motion of a two-mass system. Journal of Applied Mathematics and Mechanics, vol. 66, no. 1, p. 1-7, D0l:10.1016/S00218928(02)00002-3.

[11] Li, H., Furuta, K., Chernousko, F.L. (2006). Motion generation of the Capsubot using internal force and static friction. Proceedings of the 45th IEEE Conference on Decision and Control, p. 6575-6580, Dol:10.1109/CDC.2006.377472.

[12] Fang, H., Xu, J. (2011). Dynamics of a mobile system with an internal acceleration-controlled mass in a resistive medium. Journal of Sound and Vibration, vol. 330, no. 16, p. 40024018, D0l:10.1016/j.jsv.2011.03.010.

[13] Liu, Y., Pavlovskaia, E., Hendry, D., Wiercigroch, M. (2013). Vibro-impact responses of capsule system with various friction models. International Journal of Mechanical Sciences, vol. 72, p. 39-54, DOI:10.1016/j.ijmecsci.2013.03.009.
[14] Liu, Y., Pavlovskaia, E., Wiercigroch, M, Peng, Z. (2015). Forward and backward motion control of a vibro-impact capsule system. International Journal of Non-Linear Mechanics, vol. 70, p. 30-46, D0l:10.1016/j.jjnonlinmec.2014.10.009.

[15] Páez Chávez, J., Liu, Y., Pavlovskaia, E., Wiercigroch, M. (2016). Path-following analysis of the dynamical response of a piecewise-linear capsule system. Communications in Nonlinear Science and Numerical Simulation, vol. 37, p. 102114, DOI:10.1016/j.cnsns.2016.01.009.

[16] Liu, Y., Pavlovskaia, E., Wiercigroch, M. (2016). Experimental verification of the vibro-impact capsule model. Nonlinear Dynamics, vol. 83, no. 1, p. 1029-1041, D0l:10.1007/s11071015-2385-6.

[17] Feng, J., Huang, P.Y., Joseph, D.D. (1995). Dynamic simulation of the motion of capsules in pipelines. Journal of Fluid Mechanics, vol. 286, p. 201-227, Dol:10.1017/ S002211209500070X.

[18] Khalil, M.F., Kassab, S.Z., Adam, I.G., Samaha, M.A. (2009). Prediction of lift and drag coefficients on stationary capsule in pipeline. The $13^{\text {th }}$ International Water Technology Conference, p. 435-461.

[19] Borregales, M.A., Ensalzado, R., Asuaje, M. (2014). Prediction of lift and drag coefficients on stationary capsule in pipeline. ASME 2014 International Mechanical Engineering Congress and Exposition, p. V007T09A082, D0l:10.1115/ IMECE2014-37452.

[20] Li, W., Lu, S., Liu, Y., Wang, R., Huang, Q., Yan, J. (2015). CFD Simulation of the unsteady flow of a single coal log in a pipe. The Canadian Journal of Chemical Engineering, vol. 93, no. 11, p. 2084-2093, Dol:10.1002/cjce.22312.

[21] Botros, K.K., Golshan, H. (2010). Field validation of a dynamic model for an MFL ILI tool in gas pipelines. The $8^{\text {th }}$ International Pipeline Conference, p. 325-336, D0I:10.1115/IPC201031018. 